\title{
Gauge Symmetry and Slavnov-Taylor Identities for Randomly Stirred Fluids
}

\author{
Arjun Berera $^{1, *}$ and David Hochberg ${ }^{2, \dagger}$ \\ ${ }^{1}$ School of Physics, University of Edinburgh, Edinburgh EH9 3JZ, United Kingdom \\ ${ }^{2}$ Centro de Astrobiología (CSIC-INTA), Carretera Ajalvir Kilómetro 4, 28850 Torrejón de Ardoz, Madrid, Spain
}

(Received 7 June 2007; published 19 December 2007)

\begin{abstract}
The path integral for randomly forced incompressible fluids is shown to have an underlying BecchiRouet-Stora (BRS) symmetry as a consequence of Galilean invariance. This symmetry must be respected to have a consistent generating functional, free from both an overall infinite factor and spurious relations amongst correlation functions. We present a procedure for respecting this BRS symmetry, akin to gauge fixing in quantum field theory. Relations are derived between correlation functions of this gauge-fixed, BRS symmetric theory, analogous to the Slavnov-Taylor identities of quantum field theory.
\end{abstract}

DOI: 10.1103/PhysRevLett.99.254501

To be consistent with Newtonian physics, the description of fluid dynamics and turbulence must be the same in all inertial reference frames. For this reason, the role that Galilean invariance plays in the dynamics of a fluid system governed by the Navier-Stokes equation has been a major focus of study for decades [1,2]. The Galilean invariance of the Navier-Stokes equation plays an important part in the physical aspects of turbulence [3], in practical aspects of turbulence modeling [4], and even in fluid animation and computer graphics applications [5]. Among the symmetries of the Navier-Stokes equation, Galilean invariance is by far the most studied in theoretical approaches to turbulence $[1,2,6-9]$. The physical constraint of Galilean invariance must be taken into account in the modeling of subgrid-scale stresses in large eddy simulations [6], in models of multicomponent turbulence [8], in nonperturbative renormalization group analyses of fully developed turbulence [10], and in probability density transport equations [11]. Much of the interest has dealt with the infinite number of exact relations between different correlation functions implied by Galilean invariance, which are akin to the Ward-Takahashi identities of quantum field theory [12]. The most well known of these exact identities relates the vertex and response functions [2]. Based on this relation, various inferences have been made about the nonrenormalization of the advective or inertial term in the Navier-Stokes equation $[1,2,7,8,10,13,14]$.

In this Letter we observe that the Galilean invariant generating functional for stirred fluids $[2,15,16]$, from which all the Ward identities are derived, possesses an overall infinite factor, which must first be extracted before this functional is well defined. We draw an analogy with Abelian gauge theory in quantum field theory, whereby the Galilean invariance is associated with gauge invariance, and so the infinity arises from integrating the fluid velocity over gauge equivalent copies (i.e., over an infinite number of independent inertial reference frames) of the same theory. To remove the infinite factor in the Navier-Stokes generating functional, the Faddeev-Popov procedure of quantum field theory [17] is used to "gauge fix" the theory, that is, to fix the theory to a single inertial reference frame.
PACS numbers: 47.27.ef, 03.50.-z, 11.10.-z

The gauge fixing results in the theory no longer appearing Galilean invariant. However, we demonstrate that this gauge-fixed (GF) theory possesses an underlying BecchiRouet-Stora (BRS) symmetry $[12,18,19]$, which when invoked, restores the Galilean invariance.

This work provides a new insight into the structure of the Navier-Stokes equation. However, it is not simply a new way to express the same theory. We show that the infinite factor associated with the standard dynamic functional has associated with it much more dire consequences, in that there exist spurious relations amongst correlation functions. Explicit examples of such spurious relations are given. We then demonstrate that the gauge-fixing BRS procedure implemented here not only removes the infinite factor, but also eliminates these spurious relations, thereby rendering a well-defined generating functional for this theory. The BRS-invariant functional derived in this Letter is the consistent and correct functional for the Navier-Stokes theory that must be used to obtain reliable results.

The standard dynamic generating functional for a stirred incompressible fluid has been extensively studied for many years $[2,7,8,10,14-16]$. It is based on the path integral approach to classical statistical dynamics $[15,20]$ and is given by

$Z=\int[D \mathbf{V}][D \boldsymbol{\sigma}] \exp \left\{-S[\mathbf{V}, \boldsymbol{\sigma}]+\int d \mathbf{k} d \omega(\mathbf{J} \cdot \mathbf{V}+\boldsymbol{\Sigma} \cdot \boldsymbol{\sigma})\right\}$,

where the action

$$
\begin{aligned}
S[\mathbf{V}, \boldsymbol{\sigma}]= & \frac{1}{2} \int d \mathbf{k} d \omega \sigma_{i}(-\mathbf{k},-\omega) D_{i j}(-\mathbf{k}) \sigma_{j}(\mathbf{k}, \omega) \\
& -i \int d \mathbf{k} d \omega \sigma_{\alpha}(-\mathbf{k},-\omega) \\
& \times\left[\left(-i \omega+\nu k^{2}\right) V_{\alpha}(\mathbf{k}, \omega)-M_{\alpha \beta \gamma}(\mathbf{k})\right. \\
& \left.\times \int d \mathbf{j} d \omega_{1} V_{\beta}\left(\mathbf{k}-\mathbf{j}, \omega-\omega_{1}\right) V_{\gamma}\left(\mathbf{j}, \omega_{1}\right)\right] .
\end{aligned}
$$

The fluid velocity is $\mathbf{V}$, the conjugate field is $\boldsymbol{\sigma}, \mathbf{J}$ and $\mathbf{\Sigma}$ are $\mathbf{k}$ and $\omega$ dependent sources. $D_{i j}(-\mathbf{k})$ is the only non- 
vanishing cumulant of the random force, $M_{\alpha \beta \gamma}(\mathbf{k})=$ $(2 i)^{-1}\left\{k_{\beta} P_{\alpha \gamma}(\mathbf{k})+k_{\gamma} P_{\alpha \beta}(\mathbf{k})\right\}$ and $P_{\alpha \beta}(\mathbf{k})=\delta_{\alpha \beta}-\frac{k_{\alpha} k_{\beta}}{|\mathbf{k}|^{2}}$, and $\nu$ is the bare fluid viscosity. Throughout this Letter we will work in wave-vector frequency space.

Consider a second primed reference frame moving with respect to the unprimed frame at constant velocity $\mathbf{c}=c \hat{\mathbf{n}}$ where $\hat{\mathbf{n}}$ is a unit vector in the direction of motion. The relations between wave vector and the frequency of an event in the two frames and the transformations of the velocity and conjugate fields are given by

$$
\begin{gathered}
\mathbf{k}^{\prime}=\mathbf{k}, \quad \omega^{\prime}=\omega-\mathbf{c} \cdot \mathbf{k}, \\
\mathbf{V}(\mathbf{k}, \omega)=\mathbf{V}^{\prime}(\mathbf{k}, \omega-\mathbf{c} \cdot \mathbf{k})+\mathbf{c} \delta^{3}(\mathbf{k}) \delta(\omega), \\
\boldsymbol{\sigma}(\mathbf{k}, \omega)=\boldsymbol{\sigma}^{\prime}(\mathbf{k}, \omega-\mathbf{c} \cdot \mathbf{k}) .
\end{gathered}
$$

The action $S$ and functional measure are invariant under this Galilean transformation. The Faddeev-Popov procedure of quantum field theory $[12,17]$ is now applied to show that for $\mathbf{J}=\mathbf{\Sigma}=\mathbf{0}$, the functional in Eq. (1) has an overall infinite factor. For this, expressing the velocity zero mode as $\mathbf{V}(\mathbf{0}, 0)=\mathbf{V}_{0} \delta^{3}(\mathbf{0}) \delta(0)$ [21], first insert the factor of unity $1=\int d \mathbf{b} \delta^{3}\left(\mathbf{V}_{0}-\mathbf{b}\right)$ into Eq. (1) to give

$$
Z=\int[D \mathbf{V}][D \boldsymbol{\sigma}] \int d \mathbf{b} \delta^{3}\left(\mathbf{V}_{0}-\mathbf{b}\right) \exp \{-S[\mathbf{V}, \boldsymbol{\sigma}]\} .
$$

The observation is now made that the integrand in Eq. (6) is independent of $\mathbf{b}$. To see this, consider changing from $\mathbf{b} \rightarrow$ $\mathbf{b}+\mathbf{a}$. Then perform a Galilean transformation with boost velocity $\mathbf{c}=\mathbf{a}$ in Eqs. (3)-(5). The action and measure are invariant to this transformation and $\delta^{3}\left(\mathbf{V}_{\mathbf{0}}-(\mathbf{b}+\mathbf{a})\right) \rightarrow$ $\delta^{3}\left(\mathbf{V}_{0}-\mathbf{b}\right)$, thus restoring the integrand to its original form. Because of the independence of the integrand on the value of $\mathbf{b}$, the $d \mathbf{b}$ integral can completely factor out as an overall infinity as

$$
Z=\left(\int d \mathbf{b}\right) \int[D \mathbf{V}][D \boldsymbol{\sigma}] \delta^{3}\left(\mathbf{V}_{0}-\mathbf{b}\right) \exp \{-S[\mathbf{V}, \boldsymbol{\sigma}]\} .
$$

The term in large parentheses on the right-hand side is the infinite factor we originally sought to isolate. At this stage one could simply eliminate this integral since it is just an overall, albeit infinite, normalization factor. In this case different choices of $\mathbf{b}$ correspond to expressing the theory in different inertial reference frames. On the other hand, since the integrand in Eq. (6) is independent of $\mathbf{b}$, we can also insert any function $G(\mathbf{b})$ into the integrand, for example, to render the $\mathbf{b}$ integration finite. One convenient choice is $G(\mathbf{b})=\exp \left(-\frac{1}{2 \xi} \mathbf{b} \cdot \mathbf{b}\right)$, where $\xi>0$. Inserting this into Eq. (7) and now performing the $d \mathbf{b}$ integration, leaves the GF functional

$$
Z_{\mathrm{GF}}^{\prime}=\int[D \mathbf{V}][D \boldsymbol{\sigma}] \exp \left\{-S[\mathbf{V}, \boldsymbol{\sigma}]-\frac{1}{2 \xi} \mathbf{V}_{0} \cdot \mathbf{V}_{0}\right\} .
$$

In our analogy to gauge theories, all these different possibilities for $G(\mathbf{b})$ above would be called gauge choices.

Problems also arise if one attempts to compute any correlation function using Eq. (1). For example, consider $\left\langle V_{\alpha}(\mathbf{k}, \omega) V_{\beta}\left(\mathbf{k}^{\prime}, \omega^{\prime}\right)\right\rangle$. Using the Galilean invariance of the action, this can be written as

$$
\begin{aligned}
& \left\langle V_{\alpha}(\mathbf{k}, \omega) V_{\beta}\left(\mathbf{k}^{\prime}, \omega^{\prime}\right)\right\rangle \\
& =\frac{\int[D \tilde{\mathbf{V}}][D \boldsymbol{\sigma}] \exp \{-S[\tilde{\mathbf{V}}, \boldsymbol{\sigma}]\} \int\left[D \tilde{\mathbf{V}}_{\mathbf{c}}\right] V_{\alpha}(\mathbf{k}, \omega) V_{\beta}\left(\mathbf{k}^{\prime}, \omega^{\prime}\right)}{Z[\mathbf{0}, \mathbf{0}]},
\end{aligned}
$$

where the $[D \tilde{\mathbf{V}}]$ integration is over all velocity field configurations that are not related by Galilean invariance, and for each such field configuration the $\left[D \tilde{\mathbf{V}}_{\mathbf{c}}\right]$ integration is over all field configurations related to the particular field by a Galilean transformation. The expression Eq. (9) shows that using the ungauge-fixed functional $Z$, Eq. (1), the calculation of a correlation function sums over configurations from all Galilean-related reference frames, which in general will lead to an infinite, ill-defined quantity. This same situation would hold for a correlation function comprised of any combination of fields. Moreover, since these infinities are not just simple terms that factor out, it means they cannot be removed from the generating functional by simply dividing as $Z[\mathbf{J}, \mathbf{\Sigma}] / Z[\mathbf{0}, \mathbf{0}]$.

The gauge-fixing term in Eq. (8) manifestly breaks the Galilean invariance of the action. We next demonstrate that this gauge-fixed theory possesses a symmetry akin to the BRS symmetry of quantum field theory $[12,18,19]$, which when recognized restores the Galilean invariance of the gauge-fixed theory. We can multiply the generating functional $Z_{\mathrm{GF}}^{\prime}$ in Eq. (8) by the constant $\int d \boldsymbol{\eta} d \boldsymbol{\eta}^{*} \exp \left\{i \boldsymbol{\eta}^{*} \cdot \boldsymbol{\eta}\right\}$ where $\boldsymbol{\eta}$ and $\boldsymbol{\eta}^{*}$ are constant complex conjugate Grassmann vectors $\left\{\eta_{i}, \eta_{j}^{*}\right\}=0, \boldsymbol{\eta}^{2}=\boldsymbol{\eta}^{* 2}=0$, and are independent of $\mathbf{k}$ and $\omega$. For the " $V_{0}^{2}$ gauge" of Eq. (8) consider the following (infinitesimal) BRS transformation

$$
\begin{gathered}
\delta_{\mathrm{BRS}} \mathbf{V}_{0}=-c \zeta\left(\boldsymbol{\eta}^{*}+\boldsymbol{\eta}\right), \\
\delta_{\mathrm{BRS}} \boldsymbol{\eta}=-\frac{i}{\xi} \mathbf{V}_{0} c \zeta, \quad \delta_{\mathrm{BRS}} \boldsymbol{\eta}^{*}=+\frac{i}{\xi} \mathbf{V}_{0} c \zeta,
\end{gathered}
$$

where $c$ is a constant with dimensions of velocity, $\zeta$ is a real Grassmann parameter (independent of $\mathbf{k}$ and $\omega$ ), while all other modes of the velocity $\mathbf{V}(\mathbf{k}, \omega)$ and $\boldsymbol{\sigma}(\mathbf{k}, \omega)$ transform under $\delta_{\text {BRS }}$ as implied by Eqs. (3)-(5), except we replace the velocity boost c by $\mathbf{c} \rightarrow c \zeta\left(\boldsymbol{\eta}^{*}+\boldsymbol{\eta}\right)$. The gauge-fixed action,

$$
S_{\mathrm{GF}}\left[\mathbf{V}, \boldsymbol{\sigma}, \boldsymbol{\eta}, \boldsymbol{\eta}^{*}\right]=S[\mathbf{V}, \boldsymbol{\sigma}]+\frac{1}{2 \xi} \mathbf{V}_{0} \cdot \mathbf{V}_{0}-i \boldsymbol{\eta}^{*} \cdot \boldsymbol{\eta},
$$

is BRS invariant: $\delta_{\mathrm{BRS}} S_{\mathrm{GF}}\left[\mathbf{V}, \boldsymbol{\sigma}, \boldsymbol{\eta}, \boldsymbol{\eta}^{*}\right]=0$. In the gaugefixed functional

$$
\begin{aligned}
Z_{\mathrm{GF}}= & \int[D \mathbf{V}][D \boldsymbol{\sigma}] d \boldsymbol{\eta} d \boldsymbol{\eta}^{*} \exp \left\{-S_{\mathrm{GF}}\left[\mathbf{V}, \boldsymbol{\sigma}, \boldsymbol{\eta}, \boldsymbol{\eta}^{*}\right]\right. \\
& \left.+\int d \mathbf{k} d \omega(\mathbf{J} \cdot \mathbf{V}+\mathbf{\Sigma} \cdot \boldsymbol{\sigma})+\boldsymbol{\theta}^{*} \cdot \boldsymbol{\eta}+\boldsymbol{\theta} \cdot \boldsymbol{\eta}^{*}\right\},
\end{aligned}
$$

where $\boldsymbol{\theta}$ and $\boldsymbol{\theta}^{*}$ are constant Grassmann vector source terms; it is this BRS invariance that replaces the Galilean transformation and leads to relations amongst correlation functions, which in analogy with quantum field theory, will be called the Slavnov-Taylor identities [12,19]. 
To derive these, start with the above gauge-fixed functional $Z_{\mathrm{GF}}\left[\mathbf{J}, \boldsymbol{\Sigma}, \boldsymbol{\theta}, \boldsymbol{\theta}^{*}\right]$ and displace all fields by the infinitesimal BRS transformation Eqs. (3)-(5), with $\mathbf{c} \rightarrow c \zeta\left(\boldsymbol{\eta}^{*}+\boldsymbol{\eta}\right)$ and Eqs. (10). Since the measure and the gauge-fixed action $S_{\mathrm{GF}}$ are BRS invariant, only the source terms will be displaced. Moreover, as $\zeta^{2}=0$, we can easily expand the exponential. The gauge-fixed functional transforms as $Z_{\mathrm{GF}} \rightarrow Z_{\mathrm{GF}}+\delta_{\mathrm{BRS}} Z_{\mathrm{GF}}$, where $\delta_{\mathrm{BRS}} Z_{\mathrm{GF}}=0$ can be written as follows [21]:

$$
\left[\frac{i}{\xi}\left(\boldsymbol{\theta}^{*}-\boldsymbol{\theta}\right) \cdot \frac{\delta}{\delta \mathbf{J}_{\mathbf{0}}}+\left(\frac{\partial}{\partial \theta_{j}}+\frac{\partial}{\partial \theta_{j}^{*}}\right) \hat{O}_{j}\right] Z_{\mathrm{GF}}=0,
$$

with $\quad \hat{O}_{j}=\int d \mathbf{k} d \omega\left[J_{m}(-) k_{j} \frac{\partial}{\partial \omega} \frac{\delta}{\delta J_{m}(-)}+\Sigma_{m}(-) k_{j} \frac{\partial}{\partial \omega} \times\right.$ $\left.\frac{\delta}{\delta \Sigma_{m}(-)}-J_{j}(-) \delta(\mathbf{k}) \delta(\omega)\right]$ and $(-)$ means $(-\mathbf{k},-\omega)$.

We now show that the gauge-fixed BRS symmetric theory Eq. (11) and the resulting Slavnov-Taylor identities in Eq. (13) are not simply a different way to write the known Ward identities [2] of this theory. First we demonstrate that the standard generating functional for the Navier-Stokes equation [Eqs. (1) and (2)], in the absence of gauge fixing, contains spurious relations amongst correlation functions. To see this, return to Eqs. (1) and (2) and perform the Galilean transformations Eqs. (3)-(5). Since the measure and action $S$ are invariant under this particular change of variables, it leads to the relation

$$
\begin{aligned}
\langle & \left.\exp \int d \mathbf{k} d \omega\{\mathbf{J}(-) \cdot \mathbf{V}(\mathbf{k}, \omega)+\mathbf{\Sigma}(-) \cdot \boldsymbol{\sigma}(\mathbf{k}, \omega)\}\right\rangle \\
= & \left\langle\exp \int d \mathbf{k} d \omega\{\mathbf{J}(-) \cdot \mathbf{V}(\mathbf{k}, \omega+\mathbf{c} \cdot \mathbf{k})\right. \\
& \left.\left.-\mathbf{c} \cdot \mathbf{J}(-) \delta^{3}(\mathbf{k}) \delta(\omega)+\mathbf{\Sigma}(-) \cdot \boldsymbol{\sigma}(\mathbf{k}, \omega+\mathbf{c} \cdot \mathbf{k})\right\}\right\rangle,
\end{aligned}
$$

where the averaging on both sides is with respect to the same measure and action $S$ in Eq. (2). The above relation implies an infinite number of spurious relations. For example, acting with one derivative $\delta / \delta J_{i}(-\mathbf{k}, \omega)$ on both sides and setting $\mathbf{J}=\mathbf{\Sigma}=\mathbf{0}$, it gives $\left\langle\mathbf{V}_{i}(\mathbf{k}, \omega)\right\rangle=$ $\left\langle\mathbf{V}_{i}(\mathbf{k}, \omega+\mathbf{c} \cdot \mathbf{k})-\mathbf{c}_{i} \delta^{3}(\mathbf{k}) \delta(\omega)\right\rangle$. Integrating over an infinitesimal neighborhood near $\mathbf{k}=\mathbf{0}, \omega=0$, it leads to the relation $\mathbf{c}_{i}=0$, which is meaningless, since $\mathbf{c}$ is an arbitrary real vector which we are free to choose. Likewise, taking two derivatives $\delta / \delta J_{i}\left(-\mathbf{k}_{1}, \omega_{1}\right) \delta / \delta J_{j}\left(-\mathbf{k}_{2}, \omega_{2}\right)$ of Eq. (14) when integrated over an infinitesimal neighborhood near $\mathbf{k}_{1,2}=\mathbf{0}, \omega_{1,2}=0$ leads to the relation $-c_{j} \Delta \mathbf{k}_{1} \Delta \omega_{1}\left\langle\mathbf{V}_{i}(\mathbf{0}, 0)\right\rangle-c_{i} \Delta \mathbf{k}_{2} \Delta \omega_{2}\left\langle\mathbf{V}_{j}(\mathbf{0}, 0)\right\rangle+c_{i} c_{j}=0$, which again requires $\mathbf{c}=\mathbf{0}$, which is senseless. In a similar fashion, any number of further derivatives of $\mathbf{J}$ and/or $\mathbf{\Sigma}$ fields will lead to an infinite number of spurious relations. These examples show that at order $\mathbf{c}$ and all higher orders the generating functional Eq. (1) contains an infinite number of spurious relations. Although many papers $[7,8,10,14,16]$ have studied the generating functional Eq. (1), to our knowledge this Letter is the first to reveal this inherent inconsistency.

At $O(c)$, an alternative way to reveal spurious relations is to work directly with the Ward identity expression. For the standard generating functional, the standard Ward identity relation found in Refs. [7,8,10,14] is $\hat{O}_{j} Z=0$, where $\hat{O}_{j}$ is the operator defined after Eq. (13) and $Z$ is the generating functional Eq. (1). Consider $\delta / \delta \mathbf{J}_{i}(-\mathbf{k},-\omega) \times$ $\left.\hat{O}_{j}\right|_{\mathbf{J}=\mathbf{\Sigma}=\mathbf{0}} Z=0$, this leads to the relation $\left\langle k_{j} \frac{\partial}{\partial \omega} \mathbf{V}_{i}(\mathbf{k}, \omega)-\right.$ $\left.\delta_{i j} \delta^{3}(\mathbf{k}) \delta(\omega)\right\rangle=0$. Contract this with $\delta_{i j}$, then fluid incompressibility implies that $\delta^{3}(\mathbf{k}) \delta(\omega)=0$, which is certainly false for $\mathbf{k}=\mathbf{0}$ and $\omega=0$. However, now we can exhibit an infinite number of such spurious relations all at $O(c)$. For example, if two derivatives are taken $\delta /$ $\delta \mathbf{J}_{i}\left(-\mathbf{k}_{1},-\omega_{1}\right) \delta /\left.\delta \mathbf{J}_{m}(-\mathbf{k},-\omega) \hat{O}_{j}\right|_{\mathbf{J}=\mathbf{\Sigma}=\mathbf{0}} Z=0$, then after contraction, fluid incompressibility implies the relation $\delta^{3}\left(\mathbf{k}_{1}\right) \delta\left(\omega_{1}\right)\left\langle\mathbf{V}_{m}(\mathbf{k}, \omega)\right\rangle=0$, but the mean fluid velocity $\langle\mathbf{V}(\mathbf{k}, \omega)\rangle$ can be generally nonzero for an appropriate noise force. So, this relation is also spurious. Moreover, if one more derivative is taken with respect to $\mathbf{J}$ or $\mathbf{\Sigma}$, irrespective of the properties of the noise force, the resulting relation would be spurious. And similarly, any additional number of such derivatives will lead to an infinite number of spurious relations in which they will wrongly imply higher order velocity correlation functions are vanishing.

In contrast, for the case of our gauge-fixed generating functional Eq. (12), all spurious relations have been eliminated. At all orders higher than $O(c)$, this follows elegantly from the Grassmann property that $\zeta^{2}=0$. And at $O(c)$ the first nontrivial relation is obtained by differentiating Eq. (13) by $\delta / \delta \mathbf{J}_{0 l} \delta / \delta \theta_{j}$ to give

$$
\frac{i}{\xi}\left\langle V_{0 l} V_{0 j}\right\rangle+\left\langle\eta_{j}^{*} \eta_{l}\right\rangle=0
$$

which can be checked is correct with a similar relation found by replacing the differentiation by $\theta_{j}$ with $\theta_{j}^{*}$. Further $O(c)$ relations can be obtained by taking more $\mathbf{J}$ and/or more $\boldsymbol{\theta}$ or $\boldsymbol{\theta}^{*}$ derivatives, but due to factorization, these are all proportional to this fundamental identity Eq. (15), and so the GF-BRS functional Eq. (12) is completely free of spurious relations.

These considerations support our statement that the generating functional Eq. (12) is the correct and consistent one for this theory, and the correct expression of the Galilean invariance is the Slavnov-Taylor identities Eq. (13), found in this Letter.

Until now, we have been working with the generating functional $Z_{\mathrm{GF}}$ Eq. (12), but all our considerations can be applied to the generating functionals of connected diagrams $W=\ln Z_{\mathrm{GF}}$ and one-particle irreducible diagrams (i.e., the effective action), $\Gamma\left[\mathbf{V}_{c l}, \boldsymbol{\sigma}_{c l}, \boldsymbol{\eta}_{c l}, \boldsymbol{\eta}_{c l}^{*}\right]=$ $-W\left[\mathbf{J}, \mathbf{\Sigma}, \boldsymbol{\theta}, \boldsymbol{\theta}^{*}\right]+\boldsymbol{\theta}^{*} \cdot \boldsymbol{\eta}_{c l}+\boldsymbol{\theta} \cdot \boldsymbol{\eta}_{c l}^{*}+\int d \mathbf{k} d \omega\left(\mathbf{J} \cdot \mathbf{V}_{c l}+\right.$ $\left.\boldsymbol{\Sigma} \cdot \boldsymbol{\sigma}_{c l}\right)$. From the latter we see that $J_{k}=$ $\frac{\delta \Gamma}{\delta V_{k}^{c l}}, \Sigma_{k}=\frac{\delta \Gamma}{\delta \sigma_{k}^{c l}}, \theta_{j}=\frac{\partial \Gamma}{\partial \eta_{j}^{* c l}}, \theta_{j}^{*}=\frac{\partial \Gamma}{\partial \eta_{j}^{c l}}$.

The noise-averaged fields in the presence of the sources are denoted $\mathbf{V}_{\mathrm{cl}}=\langle\mathbf{V}\rangle_{\text {sources, }}$, etc. In terms of $\Gamma$ we can immediately write down the identity Eq. (13) in the form 


$$
\begin{aligned}
0= & \frac{i}{\xi} \mathbf{V}_{0}^{\mathrm{cl}} \cdot \frac{\partial \Gamma}{\partial \boldsymbol{\eta}_{\mathrm{cl}}}-\frac{i}{\xi} \mathbf{V}_{0}^{\mathrm{cl}} \cdot \frac{\partial \Gamma}{\partial \boldsymbol{\eta}_{\mathrm{cl}}^{*}}+\left(\eta_{\mathrm{cl}}+\eta_{\mathrm{cl}}^{*}\right)_{j} \\
& \times \int d \mathbf{k} d \omega\left(k_{j} \frac{\partial V_{m}^{\mathrm{cl}}(\mathbf{k}, \omega)}{\partial \omega} \frac{\delta \Gamma}{\delta V_{m}^{\mathrm{cl}}(\mathbf{k}, \omega)}+k_{j} \frac{\partial \sigma_{m}^{\mathrm{cl}}(\mathbf{k}, \omega)}{\partial \omega}\right. \\
& \left.\times \frac{\delta \Gamma}{\delta \sigma_{m}^{\mathrm{cl}}(\mathbf{k}, \omega)}-\delta(\mathbf{k}) \delta(\omega) \frac{\delta \Gamma}{\delta V_{j}^{\mathrm{cl}}(\mathbf{k}, \omega)}\right)
\end{aligned}
$$

We now apply this formula to the problem at hand. The dependence of $\Gamma$ on $\boldsymbol{\eta}$ and $\boldsymbol{\eta}^{*}$ is simple since these constant Grassmann vector fields do not interact nor do they couple to the velocity or conjugate fields. Thus, we can immediately write $\Gamma\left[\mathbf{V}_{\mathrm{cl}}, \boldsymbol{\sigma}_{\mathrm{cl}}, \boldsymbol{\eta}_{\mathrm{cl}}, \boldsymbol{\eta}_{\mathrm{cl}}^{*}\right]=-i \boldsymbol{\eta}_{\mathrm{cl}}^{*} \cdot \boldsymbol{\eta}_{\mathrm{cl}}+\Gamma\left[\mathbf{V}_{\mathrm{cl}}, \boldsymbol{\sigma}_{\mathrm{cl}}\right]$, where $\Gamma\left[\mathbf{V}_{\mathrm{cl}}, \boldsymbol{\sigma}_{\mathrm{cl}}\right]$ does not depend on either $\boldsymbol{\eta}_{\mathrm{cl}}$ or $\boldsymbol{\eta}_{\mathrm{cl}}^{*}$, with the first few terms written in [22].

The pertinent identity we seek is then obtained by inserting $\Gamma$ into Eq. (16) and then differentiating this with respect to $\partial / \partial \eta_{j}^{\mathrm{cl}} \delta / \delta V_{l}^{\mathrm{cl}}(\mathbf{k}, \omega) \delta / \delta \sigma_{n}^{\mathrm{cl}}(-\mathbf{k},-\omega)$ followed by setting $\boldsymbol{\eta}^{\mathrm{cl}}=\boldsymbol{\eta}_{\mathrm{cl}}^{*}=\mathbf{V}^{\mathrm{cl}}=\boldsymbol{\sigma}^{\mathrm{cl}}=0$. Note that the terms in Eq. (16) depending on the gauge parameter $\xi$ do not contribute, thus giving the identity $[7,8,10,14]$ $-k_{m} \frac{\partial}{\partial \omega} \Gamma_{l n}^{(1,1)}(-\mathbf{k},-\omega ; \mathbf{k}, \omega)=\Gamma_{m l n}^{(2,1)}(\mathbf{0}, 0 ;-\mathbf{k},-\Omega ; \mathbf{k}, \omega)$. This well known identity, has been explicitly verified up to one-loop order [1,7]. The GF-BRS theory preserves this identity as it should, but this theory is moreover free of all spurious relations. In addition, the origin of this identity is seen through the GF-BRS approach as arising in the limit of vanishing mean translational velocity, but completely independent of the properties of the fluctuating velocity. This conclusion, in agreement with [13,14], is seen here in a systematic, mathematically consistent, derivation.

The implications and consequences of Galilean invariance for Navier-Stokes fluid dynamics have been outstanding questions for some time now $[1,2,6-11,13,14]$. The formulation of the Navier-Stokes equation subject to random forcing as a classical stochastic field theory $[2,7,8,10,14-16]$ opens up the way to apply the systematic techniques originally developed for quantum fields [19]. This Letter has shown that a field theoretic approach provides clear understanding of the Galilean symmetry through its close relation to gauge fixing and BRS symmetry; the constraint singling out a unique reference frame leads to a global symmetry with anticommuting parameters first used in quantum gauge field theory [18].

In summary, we have discovered a new symmetry in Galilean invariant classical stochastic field theories, which provides a powerful new way to think about the analysis of these systems. In this Letter we focused on the NavierStokes equation but in broader terms our analysis applies also to other Galilean invariant theories such as the KPZ equation [23], magnetohydrodynamics, and the Burgers equation. An immediate and important consequence of our result is that, within the presently understood generating functionals for these theories, there are spurious relations which our gauge-fixing procedure corrects. The treatment in this Letter assumed a stochastic noise force, but formally our treatment is also applicable without the noise force. Our work, apart from now providing the mathematically consistent expression for functionals of Galilean invariant stochastic field theories, would be important in numerical and simulation calculations of these theories.

We thank W. D. McComb for discussions. Support provided to A. B. by UKPPARC and to D. H. by CSIC-INTA.

*ab@ph.ed.ac.uk

†hochbergd@inta.es

[1] D. Forster, D. R. Nelson, and M. J. Stephen, Phys. Rev. A 16, 732 (1977).

[2] C. DeDominicis and P. C. Martin, Phys. Rev. A 19, 419 (1979).

[3] W. D. McComb, The Physics of Fluid Turbulence (Oxford University Press, Oxford, 1990).

[4] S. B. Pope, Turbulent Flows (Cambridge University Press, Cambridge, 2000).

[5] M. Shah et al., in Proceedings of the Eurographics/ACM SIGGRAPH Symposium on Computer Animation, Grenoble, 2004, edited by R. Boulic and D. K. Pai (Eurographics Association, Aire-la-Ville, Switzerland, 2004), pp. 213-220.

[6] C. Speziale, J. Fluid Mech. 156, 55 (1985).

[7] E. V. Teodorovich, J. Appl. Math. Mech. 53, 340 (1989).

[8] C.-H. Mou and P. B. Weichman, Phys. Rev. E 52, 3738 (1995).

[9] A. M. Polyakov, Phys. Rev. E 52, 6183 (1995).

[10] P. Tomassini, Phys. Lett. B 411, 117 (1997).

[11] C. Tong, Phys. Fluids 15, 2073 (2003).

[12] P. Ramond, Field Theory: A Modern Primer (BenjaminCummings, Reading, MA, 1981).

[13] W. D. McComb, Phys. Rev. E 71, 037301 (2005).

[14] A. Berera and D. Hochberg, Phys. Rev. E 72, 057301 (2005).

[15] C. DeDominicis, J. Phys. (Paris), Colloq. 37, C1-247 (1976).

[16] G. L. Eyink, J. Stat. Phys. 83, 955 (1996).

[17] L. D. Fadde'ev and V. N. Popov, Phys. Lett. B 25, 29 (1967).

[18] C. Becchi, A. Rouet, and R. Stora, Phys. Lett. B 52, 344 (1974); Ann. Phys. (N.Y.) 98, 287 (1976).

[19] J. Zinn-Justin, Quantum Field Theory and Critical Phenomena (Oxford University Press, New York, 2002), 4th ed.

[20] P. C. Martin, E. D. Siggia, and H. A. Rose, Phys. Rev. A 8, 423 (1973); H. K. Janssen, Z. Phys. B 23, 377 (1976); R. Phythian, J. Phys. A 10, 777 (1977); R. D. Jensen, J. Stat. Phys. 25, 183 (1981).

[21] $\mathbf{V}(\mathbf{0}, 0)$ and $\mathbf{V}_{\mathbf{0}}$ are related by a space-time volume factor, which in a bounded box of spatial side $L$ and temporal side $T$ goes as $L^{3} T$ and for an unbounded box is $\int d^{3} x \int d t=$ $\delta^{3}(\mathbf{0}) \delta(0)$. The source $\mathbf{J}_{\mathbf{0}}=\delta^{3}(\mathbf{0}) \delta(0) \mathbf{J}(\mathbf{0}, 0)$.

[22] $\Gamma\left[\mathbf{V}^{\mathrm{cl}}, \boldsymbol{\sigma}^{\mathrm{cl}}\right]=\cdots \int d \mathbf{q}_{1} d \omega_{1} \int d \mathbf{q}_{2} d \omega_{2} V_{\alpha}^{\mathrm{cl}}\left(\mathbf{q}_{1}, \omega_{1}\right) \times$ $\sigma_{\beta}^{\mathrm{cl}}\left(\mathbf{q}_{2}, \omega_{2}\right) \Gamma_{\alpha \beta}^{(1,1)}\left(-\mathbf{q}_{1},-\omega_{1} ;-\mathbf{q}_{2},-\omega_{2}\right)+\frac{1}{2} \int d \mathbf{q}_{1} d \omega_{1} \times$ $\int d \mathbf{q}_{2} d \omega_{2} \int d \mathbf{q}_{3} d \omega_{3} V_{\alpha}^{\mathrm{cl}}\left(\mathbf{q}_{1}, \omega_{1}\right) V_{\beta}^{\mathrm{cl}}\left(\mathbf{q}_{2}, \omega_{2}\right) \sigma_{\gamma}^{\mathrm{cl}}\left(\mathbf{q}_{3}, \omega_{3}\right) \times$ $\Gamma_{\alpha \beta \gamma}^{(2,1)}\left(-\mathbf{q}_{1},-\omega_{1} ;-\mathbf{q}_{2},-\omega_{2} ;-\mathbf{q}_{3},-\omega_{3}\right)+\cdots$.

[23] M. Kardar, G. Parisi, and Y.-C. Zhang, Phys. Rev. Lett. 58, 2087 (1987). 